\title{
ANOMALIES DES SECRETIONS DES VESICULES SEMINALES ET FECONDATION IN VITRO.
}

\author{
E. SZERMAN-JOLY*, N. LEPORRIER**, D. HERLICOVIEZ**, M. HERLICOVIEZ ${ }^{\circ}$, \\ A. SAUVALLE**, I. DENIS*, J.IZARD** et M. A. DROSDOWSKY*. \\ Laboratoire de Biochimie* et de Cytologie génitale** CHU Côte de Nacre, \\ Service de Gynécologie Obstétrique ${ }^{\circ}$ CHU Clémenceau - 14033 CAEN CEDEX
}

\begin{abstract}
ABNORMAL SEMINAL VESICLE SECRETION AND IN VITRO FERTILIZATION.

We have examined the IVF results in couples in which the husband had low fructose level as the only abnormal semen parameter. Oocyte cleavage level and pregnancy rate were not different in this group when compared to the overall population. A sperm factor responsible for the oocyte cleavage failure was found in only three couples out of seventeen. It was not possible to define its caracteristics. Key words : In vitro fertilization, fructose, seminal vesicles. Andrologie, 1991, 1 : 66.
\end{abstract}

\section{INTRODUCTION}

Les vésicules séminales (vs) comme les autres glandes du tractus génital élaborent des substances qui influencent les propriétés fonctionnelles des spermatozoïdes humains. Des altérations de leur qualité peuvent affecter la fertilité masculine (1); en effet, l'ablation des vs chez les mammifères entraine une réduction importante de la fertilité (3). Les vs secrètent du fructose qui est un bon marqueur de leur activité (2). Aussi en utilisant ce dosage ainsi qu'un test biologique direct sous la forme de fécondation in vitro (FIV) des ovocytes humains, nous avons voulu étudier l'incidence d'une anomalie dans la sécrétion des vs sur la fécondance des spermatozoïdes.

\section{MATERIELS ET METHODES}

17 couples ont été sélectionnés pour cette étude dont 13 bénéficiaient d'une FIV pour stérilité tubaire et 4 étaient adressés pour stérilité inexpliquée. Les bilans spermiologiques complets demandés à chaque patient avant constitution du dossier étaient normaux en ce concerne les spermogramme et spermocytogramme (numération $>20$ millions/ml, mobilité et formes typiques $>35 \%$ ). La recherche d'anticorps anti-spermatozoïdes était négative. Le taux de fructose dans l'éjaculat total était abaissé à 2 reprises (< 15 uM/éjaculat) (4) sans anomalie prostatique associée, c'est-à-dire des taux de citrate $\mathrm{Zn}$ et $\mathrm{Mg}$ normaux. Avant chaque tentative de FIV, une étude bactériologique du sperme a été demandée à chaque patient. 29 tentatives de FIV ont été réalisées chez ces 17 couples soit 1 à 4 tentatives par couple.
Les ovocytes non fécondés ont été analysés sur le plan cytogénétique dans les ponctions avec échec total de segmentation.

\section{RESULTATS}

Associé au taux de fructose bas, nous avons constaté d'autres anomalies du sperme ; un volume inférieur à $1,5 \mathrm{ml}$ chez 11 patients parmi les 17 avec viscosité augmentée 2 fois et mobilité secondaire diminuée 3 fois. L'analyse bactériologique s'est avérée positive pour 13 hommes, ce qui représente une incidence de $80 \%$ alors qu'elle est inférieure à $10 \%$ sur l'ensemble des couples rentrant dans un protocole de FIV. Les résultats de FIV chez ces couples traités par antibiothérapie, 2 à 3 semaines avant la tentative sont comparés à la totalité des FIV pratiquées pendant la même période (tableau 1). Le taux de segmentation des ovocytes est globalement identique dans les deux groupes, $44 \%$ vs $45 \%$. Nous avons obtenu 9 grossesses dans le groupe de fructose bas pour des pathologies féminines exclusivement tubaires, soit $31 \%$ par tentative et 142 sur l'ensemble de la population soit $16,5 \%$ par tentative. (test $\mathrm{X}_{2}$ non significatif).

Tableau 1 : RESULTATS DES FECONDATIONS IN VITRO

\begin{tabular}{lcccc}
\hline & $\begin{array}{c}\mathrm{n} \\
\text { Couples }\end{array}$ & $\begin{array}{c}\mathrm{n} \\
\text { Tentatives }\end{array}$ & $\begin{array}{c}\text { Taux de } \\
\text { segment. (\%) }\end{array}$ & $\begin{array}{c}\text { Grossesses } \\
\text { cliniques }\end{array}$ \\
\hline Fructose $<15 \mathrm{uM}$ & 17 & 29 & $44+31$ & 9 \\
Fructose $>15 \mathrm{uM}$ & 305 & 861 & $\mathbf{4 5}+37$ & 142 \\
\hline
\end{tabular}

Parmi les 8 femmes n'ayant pas eu de grossesse, 4 ont présenté un échec de transfert embryonnaire et 4 un échec de segmentation à une ou deux reprises. Ce groupe est analysé dans le tableau 2.

\section{Tableau 2: ANALYSE DES ECHECS DE SEGMENTATION}

\begin{tabular}{|c|c|c|c|c|c|}
\hline Indic & $\begin{array}{l}\text { Volume } \\
\text { Sperme }\end{array}$ & $\begin{array}{c}\text { Taux } \\
\text { Fructose } \\
\text { (uM/éjaculat) }\end{array}$ & $\begin{array}{l}\text { Bactério- } \\
\text { Sperme }\end{array}$ & $\begin{array}{c}\text { Ovocytes } \\
\text { n }\end{array}$ & $\begin{array}{l}\text { Cytogénétique } \\
\text { (meta II) n }\end{array}$ \\
\hline $\mathrm{BOU}(1)$ ? & & 10 & + & 6 & 3 \\
\hline $\operatorname{BOU}(2) ?$ & & 6 & + & 2 & 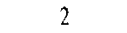 \\
\hline BLA & N & 3,5 & . & 6 & non fait \\
\hline LEB & $\mathrm{N}$ & 8 & + & 15 & 9 \\
\hline SOR(D) ? & & 5 & + & 10 & non fait \\
\hline $\operatorname{SOR}(2) ?$ & & 5,7 & - & II & 1 \\
\hline
\end{tabular}

$?:$ Indication inexpliquée - $\mathrm{T}:$ Indication tubaire

: Volume spermatique abaissé - $\mathrm{N}$ : Volume spermatique normal

Il s'agissait de 3 stérilités féminines inexpliquées et 1 stérilité tubaire. Le volume spermatique était abaissé chez 2 patients et l'analyse bactériologique positive avant 4 des 6 tentatives. Le taux de fructose n'était jamais nul. Excepté pour le couple BLA, l'analyse cytogénétique des ovocytes non fécondés a montré une majorité d'ovocytes mûrs (en métaphase II) suggérant l'existence d'un facteur masculin prépondérant dans ce groupe.

\section{DISCUSSION}

Dans cette étude, nous avons constaté qu'un taux de fructose abaissé est souvent associé à un faible volume de l'éjaculat, correspondant sans doute à de petites vs ou à une obstruction partielle, sans incidence sur la fertilité. Cette anomalie est également corrélée à une pathologie infectieuse des vs non associée à une prostatite car les marqueurs prostatiques sont normaux. Après traitement antibiotique, le taux de grossesse par FIV n'est pas modifié dans cette pathologie. Les échecs de segmentation malgré l'existence d'ovocytes fécondables sont apparus dans un groupe d'individus ne présentant pas d'anomalies spécifiquement différentes des vs par rapport aux autres patients. Le dosage de fructose apparait comme marqueur nécessaire à l'exploration des vs, mais i] serait utile de rechercher d'autres éléments permettant d'évaluer l'activité fonctionnelle des glandes.

\section{REFERENCES}

1 - Clavert A., Gabriel-Robez O., Montagnon D., Physiological role of seminal vesicle. Prog. Reprod. Biol. Med. 1985, 12, 80-94.

2 - Mann T. \& Lutwak-Mann C., Biochemistry of seminal plasma and male accessory fluids in male reproductive function \& semen. 1981. Springer verlag Berlin.

3 - Pang SF., Chow PM, Wrong TM, The role of the seminal vesicles, coagulating glands and prostate glands on the fertility and fecundity of mice. J. Reprod. Fertil., 1979, 56, 129-132.

4 - Soufir JC, Exploration biochimique du sperme humain Médecine de la reproduction masculine. (Eds G. Schaison - P. Bouchard-J. Mahoudeau-F. Labrie) Flammarion Médecine /Sciences - Presse de l'Université de Montréal. 1984, 69-74.

RESUME : Nous avons examiné les résultats de FIV pour des couples où l'homme présentait comme seule anomalie du sperme un faible taux de fructose. Le taux de segmentation des ovocyles tout comme le taux de grossesse n'est pas différent dans ce groupe comparé à l'ensemble de la population. Un facteur spermatique responsable de l'échec de segmentation des ovocytes n'a pu être démontré que pour trois couples sur les 17 sélectionnés sans possibilité d'en définir les caractéristiques. Mots clés : Fécondation in vitro, fructose, vésicules séminales. Andrologie 1991, 1 : 66. 